\title{
The relationship between chitotriosidase activity and tuberculosis
}

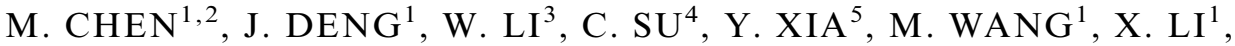 \\ B. K. ABUAKU ${ }^{1,6}, H$. TAN ${ }^{1 *}$ AND S. W. WEN ${ }^{1,7}$ \\ ${ }^{1}$ Department of Epidemiology and Health Statistics, School of Public Health, Central South University, \\ Changsha, Hunan, P. R. China \\ ${ }^{2}$ Hunan Children's Hospital, Changsha, Hunan, P.R. China \\ ${ }^{3}$ Department of Nursing, Shaoyang Medical College, Shaoyang, Hunan, P.R. China \\ ${ }^{4}$ Yueyanglou Center for Disease Control and Prevention, Yueyang, Hunan, P. R. China \\ ${ }^{5}$ Zixing Center for Disease Control and Prevention, Chengzhou, Hunan, P. R. China \\ ${ }^{6}$ Department of Epidemiology, Noguchi Memorial Institute for Medical Research, College of Health Sciences, \\ University of Ghana, Legon, Accra, Ghana \\ ${ }^{7}$ Department of Obstetrics \& Gynecology and Department of Epidemiology \& Community Medicine, University \\ of Ottawa. The Ottawa Hospital, Ottawa, Ontario Canada
}

Received 28 August 2014; Final revision 21 February 2015; Accepted 10 March 2015; first published online 1 April 2015

\section{SUMMARY}

Chitotriosidase, secreted by activated macrophages, is a biomarker of activated macrophages. In this study, we explored whether chitotriosidase could be adopted as a biomarker to evaluate the curative effect on tuberculosis (TB). Five counties were randomly selected out of 122 counties/ cities/districts in Hunan Province, China. Our cases were all TB patients who were newly diagnosed or had been receiving treatment at the Centers for Disease Control (CDCs) of these five counties between April and August in 2009. Healthy controls were selected from a community health facility in the Kaifu district of Changsha City after frequency-matching of gender and age with the cases. Chitotriosidase activity was evaluated by a fluorometric assay. Categorical variables were analysed with the $\chi^{2}$ test. Measurement data in multiple groups were tested with analysis of variance and least significant difference (LSD). Correlation between chitotriosidase activity and the degree of radiological extent (DRE) was examined by Spearman's rank correlation test. The average chitotriosidase activity levels of new TB cases, TB cases with different periods of treatment ( $<3,3-6,>6$ months) and the control group were $54 \cdot 47,34 \cdot 77$, $21.54,12.73$ and $10.53 \mathrm{nmol} / \mathrm{h} . \mathrm{ml}$, respectively. Chitotriosidase activity in TB patients declined along with the continuity of treatment. The chitotriosidase activity of both smear-positive and the smear-negative pulmonary TB patients decreased after 6 months' treatment to normal levels $(P<0 \cdot 05)$. Moreover, chitotriosidase activity was positively correlated with DRE $(r=0 \cdot 607, P<$ $0 \cdot 001)$. Our results indicate that chitotriosidase might be a marker of TB treatment effects. However, further follow-up study of TB patients is needed in the future.

Key words: Chitotriosidase, tuberculosis.

\footnotetext{
* Author for correspondence: Dr Hongzhuan Tan, School of Public Health, Central South University, Changsha, Hunan, 410008, China. (Email: tanhz99@qq.com)

This is an Open Access article, distributed under the terms of the Creative Commons Attribution-NonCommercial-NoDerivatives licence (http://creativecommons.org/licenses/by-nc-nd/3.0/), which permits non-commercial re-use, distribution, and reproduction in any medium, provided the original work is unaltered and is properly cited. The written permission of Cambridge University Press must be obtained for commercial re-use or in order to create a derivative work.
} 


\section{INTRODUCTION}

Pulmonary tuberculosis (TB) is a widespread debilitating disease that threatens public health throughout the world. It is estimated that one third of the world's population live with a latent Mycobacterium tuberculosis infection, which problematically presents a potential source of active TB in the future [1]. About $5-10 \%$ of individuals infected by $M$. tuberculosis will progress to active TB during their lifetime [2, 3]. In 2012, 8.6 million people were diagnosed with active TB worldwide, 1.3 million of whom died from the disease [4]. Of the 8.6 million TB cases, 450000 were multidrug-resistant TB (MDR-TB) and 150000 patients died from MDR-TB. China carries the second largest TB burden in the world. According to the 5th Nationwide TB Epidemiological Sampling Survey in 2010 [5], the prevalence of active and smearpositive TB were $459 / 100000$ and $66 / 100000$, respectively, in people aged $\geqslant 15$ years.

After implementation of the directly observed treatment strategy (DOTS), identification, diagnosis and treatment of TB have been increasingly standardized. To reduce the occurrence of MDR-TB, the World Health Organization (WHO) adjusted the pulmonary TB treatment plan in 2010. In the adjusted plan, it is recommended that new patients with pulmonary $\mathrm{TB}$ receive a treatment regimen with 6 months of rifampicin: 2HRZE/4HR [6]. During the long process of TB treatment, no efficient method is available to evaluate the curative effects with precision, especially for smear-negative TB, which accounts for a majority (85.6\% in China) of all TB cases. Therefore, it is crucial to find a method to more precisely evaluate the curative effects of TB treatment.

Chitotriosidase, secreted by activated macrophages [7-10], is involved in the mechanism of innate host defence against fungal infection [11]. Tuberculosis bacillus is a type of intracellular bacterium mainly parasitized in macrophages. Alveolar macrophages secrete chitotriosidase after being activated by the M. tuberculosis residing in alveolar macrophages. The chitotriosidase activity of plasma in TB patients was first investigated in 2007 [12]. However, the result of the study was not significant, which could be attributed to the small sample size. As reported, chitotriosidase activity of smear-negative culture-positive TB cases is higher compared to non-active TB patients and healthy controls [13]. The chitotriosidase level in patients with 6 months' anti-TB treatment significantly decreased to a level that was close to healthy controls [14]. These studies suggest that chitotriosidase may serve as a biomarker in evaluating the curative effects of TB treatment. Therefore, the aim of our study was to explore the difference in chitotriosidase activity in TB patients at different treatment stages and to investigate the relationship between chitotriosidase and radiological parameters.

\section{METHODS}

\section{Selection of cases}

Sources of cases: Cases were randomly selected with a two-step stratified sampling method. First, five counties out of 122 counties/cities/districts in Hunan Province were randomly selected using a random number table (e.g. Qidong county, Yueyanglou district, Yueyang county, Zixing city, Hongjiang city). According to the TB management regulation, all suspected TB patients would be transferred to local Centers for Disease Control (CDCs) for confirmation based on WHO diagnostic criteria [15]. Once confirmed, the patients would receive treatment at the CDCs free of charge. Therefore, we identified cases from local CDCs. All patients who were newly diagnosed or had been receiving TB treatment at the CDCs of these five counties between April and August 2009 were selected. Patients with HIV/TB co-infection or other co-infections including fungi and nematodes were excluded because these co-infections could enhance serum chitotriosidase activity. Patients who stopped receiving medications or were previously treated were also excluded.

\section{Selection of healthy controls}

Controls were also selected using the stratified sampling strategy. First, one from the 14 community health service centres (e.g. that in Xingang community) in Kaifu district, Changsha city was selected using the random number table. Next, one from the six community health service stations (e.g. Xin'ansi) managed by Xingang Community Health Service Centre was randomly selected. Next, controls were matched with cases using a frequency-matching method. Because the ratio of male to female TB patients was about $2 \cdot 5: 1$ in Hunan [16], the healthy controls were selected from permanent residents in Xin'ansi community by a gender-age frequencymatching method. All controls were confirmed free from active TB. 
We also excluded participants (both cases and controls) who were not Han nationality.

\section{Estimation of sample size}

Sample size was calculated with:

$n_{i j}=\frac{\left(Z_{a / 2}+Z_{1-\beta}\right)^{2} \times\left(\sigma_{2}^{2}+\sigma_{2}^{2}\right)}{\delta_{i j}^{2}}$.

The standard deviation (S.D.) $\sigma$ in controls and the $>6$-month treatment group was estimated to be $4 \cdot 2$ [14]; assuming a difference between these two groups of $\delta=2, \alpha=0 \cdot 05$ (two-sided), and $\beta=0 \cdot 10$, the estimated sample size was 93 , i.e. at least 93 participants of each group eligible for inclusion.

The protocol of our study was approved by the Ethics Review Committee of Central South University. A written informed consent form was signed by all participants. After that, $5 \mathrm{ml}$ of blood was collected through venepuncture using an EDTA-containing tube. Plasma and packed cells were separated by centrifugation at $2500 \mathrm{~g}$ for 10 min and stored at $-80^{\circ} \mathrm{C}$ until used. In addition, all TB patients underwent a chest X-ray examination to determine the degree of radiological extent (DRE). The healthy controls received free physical examinations, including chest $\mathrm{X}$-ray.

\section{Determination of DRE}

DRE was determined through chest X-rays by two chest physicians independently. In case of inconsistent interpretations, a final decision was made by consensus after the physicians evaluated the chest X-ray together. A previously described scoring system with modification was used [14, 17]. Briefly, in each chest X-ray radiograph, each lung was divided into three zones: the middle zone was set between the levels of the second and fourth ribs at the costosternal junction, the upper zone was above the middle zone, and the lower zone was below the middle zone. A chest X-ray score was determined by visually estimating the extent of the opacities attributable to TB (e.g. nodular infiltration, consolidation, atelectasis, or linear density) and separately evaluating the existence of cavitation. The scoring was based on the percentage of opacities in each lung zone $(P E R)$ and the total diameter of the cavities $(D)$. The scoring system was set as follows:

Score 0: no abnormality;

Score 1: PER of $<25 \%$ without obvious cavitation;
Score 2: PER of $25-50 \%$ without obvious cavitation, or $P E R<25 \%$ and $D<2 \mathrm{~cm}$;

Score 3: $P E R$ of $50-75 \%$ without obvious cavitation, or $P E R<50 \%$ and $D=2-4 \mathrm{~cm}$;

Score 4: $P E R>75 \%$, or any cavitary disease with $D>$ $4 \mathrm{~cm}$.

\section{Treatment regimen}

The medications used for TB treatment included isoniazid $(\mathrm{H})$, rifampicin $(\mathrm{R})$, pyrazinamide $(\mathrm{Z})$, ethambutol (E), thioacetazone (T), and streptomycin (S). The treatment regimens used in Hunan in 2009 were based on the WHO-recommended regimen [15]. Specifically, the smear-positive patients were treated with 3 times/week HRZE for the first 2 months and 3 times/week HR for 4 months (2H3R3Z3E3/4H3R3). The smear-negative patients were treated similarly except that E was not used (2H3R3Z3/4H3R3).

\section{Detection of chitotriosidase activity}

We adopted a published method for measurement of chitotriosidase activity [18]. Specifically, $5 \mu$ l of EDTA plasma was incubated with $100 \mu \mathrm{l}$ of $0.022 \mathrm{~mm}$ 4-methylumbelliferyl-fl-D- $N N, N$ '-triacetylchitotriose (4 MU-chitotrioside; Sigma Chemical Co., USA) as substrate in a citrate/phosphate buffer $(0 \cdot 1 / 0 \cdot 2 \mathrm{~m}), \mathrm{pH}$ $5 \cdot 2$, at $37^{\circ} \mathrm{C}$. After $60 \mathrm{~min}$, the reaction was stopped with addition of $0.3 \mathrm{M}$ glycine/ $\mathrm{NaOH}$ buffer $(2 \mathrm{ml}, \mathrm{pH}$ 10.6). The fluorescence intensity was tested at an excitation wavelength of $360 \mathrm{~nm}$ and emission wavelength of $450 \mathrm{~nm}$. The unit of chitotriosidase activity is 'nmol/h.ml'.

\section{Statistical analysis}

Data were analysed with SPSS v. 19.0 (IBM Corporation, USA). Categorical variables were analysed by $\chi^{2}$ test. Measurement data in multiple groups were tested with analysis of variance (ANOVA) and least significant difference (LSD). Correlation between chitotriosidase activity and DRE was examined by Spearman's rank correlation test, with the correlation coefficient expressed as $r$. All tests of hypothesis were two-tailed with a type-1 error at $5 \%$.

\section{Ethical standards}

The authors assert that all procedures contributing to this work comply with the ethical standards of the 
Table 1. Distribution of gender and age of the study population

\begin{tabular}{|c|c|c|c|c|c|c|c|}
\hline & $\begin{array}{l}\text { New TB cases } \\
n(\%)\end{array}$ & $\begin{array}{l}\text { Treatment } \\
<3 \text { months } \\
n(\%)\end{array}$ & $\begin{array}{l}\text { Treatment } \\
3-6 \text { months } \\
n(\%)\end{array}$ & $\begin{array}{l}\text { Treatment } \\
>6 \text { months } \\
n(\%)\end{array}$ & $\begin{array}{l}\text { Control } \\
n(\%)\end{array}$ & $\chi^{2}$ & $P$ \\
\hline \multicolumn{8}{|l|}{ Gender } \\
\hline Male & $190(73 \cdot 64)$ & $103(72 \cdot 03)$ & $75(73 \cdot 53)$ & $70(71 \cdot 43)$ & $67(71 \cdot 28)$ & \multirow[t]{2}{*}{0.359} & \multirow[t]{2}{*}{0.986} \\
\hline Female & $68(26 \cdot 36)$ & $40(27 \cdot 97)$ & $27(26 \cdot 47)$ & $28(28 \cdot 57)$ & $27(28 \cdot 72)$ & & \\
\hline \multicolumn{8}{|c|}{ Age group, years } \\
\hline 19-30 & $35(13 \cdot 57)$ & $21(14 \cdot 69)$ & $12(11 \cdot 76)$ & $12(12 \cdot 24)$ & $14(14 \cdot 89)$ & \multirow[t]{4}{*}{6.986} & \multirow[t]{4}{*}{$0 \cdot 861$} \\
\hline $31-50$ & $96(37 \cdot 21)$ & $56(39 \cdot 16)$ & $38(37 \cdot 25)$ & $32(32 \cdot 6)$ & $36(38 \cdot 30)$ & & \\
\hline $51-70$ & $92(35 \cdot 66)$ & $39(27 \cdot 27)$ & $36(35 \cdot 29)$ & $40(40 \cdot 82)$ & $32(34 \cdot 04)$ & & \\
\hline $71-84$ & $35(13 \cdot 57)$ & $27(18 \cdot 88)$ & $16(15 \cdot 69)$ & $14(14 \cdot 29)$ & $12(12 \cdot 77)$ & & \\
\hline
\end{tabular}

relevant national and institutional committees on human experimentation and with the Helsinki Declaration of 1975, as revised in 2008.

\section{RESULTS}

\section{Basic information of the study population}

A total of 695 participants were involved in our study, including 601 cases and 94 healthy controls (control group). The cases were at different treatment stages: new $(n=258$, case group 1), $<3$ months (143, case group 2), 3-6 months (102, case group 3), and $>6$ months (98, case group 4). The age of the study population ranged from 19 to 84 years. There was no significant difference in gender $(P=0.986)$ or age $(P=$ $0 \cdot 861)$ between cases and controls (Table 1).

\section{Chitotriosidase activity}

\section{Comparison between case groups and control group}

The average levels of plasma chitotriosidase activity in the four case groups and the control group were 54.47 $\pm 19 \cdot 27,34 \cdot 77 \pm 8 \cdot 67,21 \cdot 54 \pm 7 \cdot 56,12 \cdot 73 \pm 3.99$ and $10.53 \pm 3.47 \mathrm{nmol} / \mathrm{h} . \mathrm{ml}$, respectively. The ANOVA revealed significant difference in chitotriosidase activity $(P<0 \cdot 001)$. Chitotriosidase activity in the pulmonary TB patients declined with continuity of treatment. After 6 months' anti-TB treatment, chitotriosidase activity in both smear-positive and smearnegative patients significantly decreased to levels which were close to the healthy controls (Table 2).

\section{Correlation between chitotriosidase activity and DRE}

The consistency of DRE interpretations by the two chest physicians was satisfactory (kappa $=0.908$, Table 3). Serum chitotriosidase activity is moderately and significantly correlated with DRE $(r=0 \cdot 607, P<$ 0.001; Fig. 1, Table 4).

\section{DISCUSSION}

Plasma chitotriosidase activity levels in the four case groups and the control group were 54.47, 34.77, $21.54,12.73$ and $10.53 \mathrm{nmol} / \mathrm{h} . \mathrm{ml}$, respectively. As the treatment continued, the chitotriosidase activity of TB patients gradually declined. Chitotriosidase activity levels of both smear-positive and smear-negative patients decreased after 6 months' treatment and approached normal levels. Moreover, chitotriosidase activity is positively correlated with DRE $(r=0.607$, $P<0 \cdot 001$ ), suggesting that worse DREs correspond to higher chitotriosidase activity. Our results, as well as a previous study [14], suggest that chitotriosidase is capable of reflecting the curative effects of $\mathrm{TB}$ treatment.

In humans, alveolar macrophages can be activated by $M$. tuberculosis parasitizing inside and this activation can trigger macrophages to secrete chitotriosidase [9, 19]. Moreover, activated macrophages play an important role in the formation of tuberculous granulomas [20]. Meanwhile, the activated macrophages can promote the secretion of interferon gamma as well as tumuor necrosis factor alpha, which will indirectly enhance the secretion of chitotriosidase [7, 8]. However, it is still unclear how chitotriosidase functions physiologically in human tissue. Nevertheless, it has been widely accepted that chitotriosidase is a biomarker of macrophages, it is also a biomarker of resistance of the host against the infection of chitin-related pathogen [21-23]. The improvement of chitotriosidase activity in TB patients and its correlation with DRE suggest that chitotriosidase is possibly involved in the immunopathogenesis of 
Table 2. Chitotriosidase activity of the study groups

\begin{tabular}{|c|c|c|c|c|c|c|}
\hline & \multicolumn{6}{|c|}{ Chitotriosidase activity (nmol/h.ml) } \\
\hline & $n$ & Mean & S.D. & Min-max & $F$ & $P$ \\
\hline \multicolumn{7}{|l|}{ Total } \\
\hline New TB case & 258 & $54 \cdot 47$ & $19 \cdot 27$ & $14 \cdot 59-137 \cdot 16$ & $331 \cdot 614$ & $<0 \cdot 001$ \\
\hline Treatment $<3$ months & 143 & $34 \cdot 77 *$ & $8 \cdot 67$ & $12 \cdot 50-81 \cdot 60$ & & \\
\hline Treatment 3-6 months & 102 & $21 \cdot 54^{* \dagger}$ & $7 \cdot 56$ & $6 \cdot 85-46 \cdot 78$ & & \\
\hline Treatment $>6$ months & 98 & $12 \cdot 73^{*+t}+$ & $3 \cdot 99$ & $5 \cdot 03-20 \cdot 53$ & & \\
\hline Control & 94 & $10 \cdot 53 *+t$ & $3 \cdot 47$ & $3 \cdot 26-20 \cdot 83$ & & \\
\hline \multicolumn{7}{|l|}{ Smear positive } \\
\hline New TB case & 42 & $69 \cdot 44$ & $30 \cdot 89$ & $15 \cdot 60-137 \cdot 16$ & $110 \cdot 507$ & $<0 \cdot 001$ \\
\hline Treatment $<3$ months & 26 & $40 \cdot 10^{*}$ & $13 \cdot 19$ & $16 \cdot 93-8160$ & & \\
\hline Treatment $3-6$ months & 17 & $30 \cdot 04 * \dagger$ & $9 \cdot 88$ & $11 \cdot 69-46 \cdot 78$ & & \\
\hline Treatment $>6$ months & 18 & $17 \cdot 05^{*}+t$ & $2 \cdot 49$ & $12 \cdot 30-20 \cdot 52$ & & \\
\hline Control & 94 & $10 \cdot 53 *+t$ & $3 \cdot 47$ & $3 \cdot 2-20 \cdot 83$ & & \\
\hline \multicolumn{7}{|l|}{ Smear negative } \\
\hline New TB case & 216 & $51 \cdot 56$ & $14 \cdot 47$ & $14 \cdot 59-83 \cdot 99$ & $454 \cdot 897$ & $<0 \cdot 001$ \\
\hline Treatment $<3$ months & 117 & $33 \cdot 59 *$ & $6 \cdot 83$ & $12 \cdot 50-68 \cdot 67$ & & \\
\hline Treatment $3-6$ months & 85 & $19 \cdot 83^{* \dagger}$ & $5 \cdot 70$ & $6 \cdot 85-3 \cdot 50$ & & \\
\hline Treatment $>6$ months & 80 & $11 \cdot 76^{*+t} t$ & $3 \cdot 62$ & $5 \cdot 03-20 \cdot 38$ & & \\
\hline Control & 94 & $10 \cdot 53^{*+t}$ & $3 \cdot 47$ & $3 \cdot 2-20 \cdot 83$ & & \\
\hline
\end{tabular}

* The least significant difference (LSD) pairwise comparison showed statistical significance compared with the new case group.

$\dagger$ The LSD pairwise comparison showed statistical significance compared with the treatment $<3$ months group.

$\$$ The LSD pairwise comparison showed statistical significance compared with the treatment 3-6 months group.

Table 3. The inter-rater correlation of the degree of radiological extent (DRE) interpretation

\begin{tabular}{cllllll}
\hline \hline \multicolumn{5}{c}{ Physician B } & \\
\cline { 2 - 5 } DRE & 0 & 1 & 2 & 3 & 4 & Total \\
\hline Physician A & & & & & & \\
0 & 94 & 3 & 0 & 0 & 0 & 97 \\
1 & 4 & 52 & 5 & 0 & 0 & 61 \\
2 & 0 & 7 & 214 & 10 & 0 & 231 \\
3 & 0 & 0 & 11 & 191 & 2 & 204 \\
4 & 0 & 0 & 0 & 6 & 96 & 102 \\
Total & 98 & 62 & 230 & 207 & 98 & 695 \\
\hline \hline
\end{tabular}

Kappa $=0 \cdot 908$.

TB [14]. As reported, chitotriosidase activity of sarcoidosis patients was weakened with the remission of clinical symptoms, and was enhanced as the chest $\mathrm{X}$-ray worsened [24]. This evidence indicates that chitotriosidase activity is very likely to be a biomarker in evaluating the curative effects of anti-TB treatment.

In 2007, Bargagli et al. [12] first analysed plasma chitotriosidase activity in TB patients. The difference between TB patients and controls was not significant because of the small sample size. However, chitotriosidase activity of TB pleural effusions was significantly higher than non-TB lymphocytic pleural effusions [25]. Serum chitotriosidase levels in 17 smearnegative, culture-positive TB cases were significantly higher than in 38 smear-negative, culture-negative TB cases and 20 healthy controls $(68.05,29.73$ and 28.4 nmol/h.ml, respectively) [13]. A follow-up study [14] shows that the average chitotriosidase activity of $42 \mathrm{~TB}$ patients is significantly higher compared to 30 controls $(39.73 \pm 24.97$ vs. $9.63 \pm 4.55 \mathrm{nmol} / \mathrm{h} . \mathrm{ml}$, $P<0 \cdot 001)$. After 6 months' anti-TB treatment, chitotriosidase levels significantly decreased and approached the levels of healthy controls. At present, TB treatment effectiveness is evaluated by clinical symptoms and chest X-rays, which are strongly subjective and therefore unsatisfactory. The aforementioned evidence and our results imply that chitotriosidase might be a promising marker to evaluate the curative effects of TB treatment.

There are some limitations to our study. First, a cross-sectional study was designed to enrol cases at different treatment stages. Our study result just modestly suggests that chitotriosidase activity would be weakened as the treatment continued. However, whether chitotriosidase could serve as a marker to 


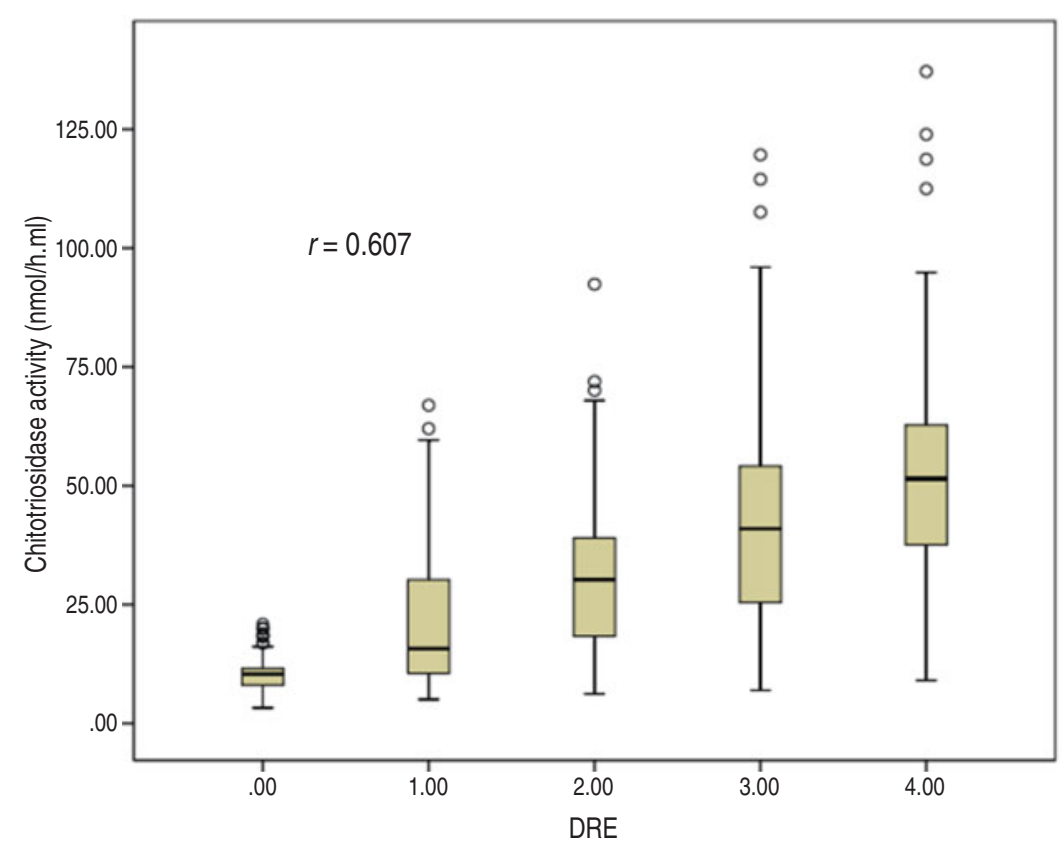

Fig. 1. Correlation between chitotriosidase activity and the degree of radiological extent (DRE).

Table 4. Chitotriosidase activity in groups with different degree of radiological extent (DRE)

\begin{tabular}{|c|c|c|c|c|c|c|}
\hline \multirow[b]{2}{*}{ DRE } & \multicolumn{4}{|c|}{ Chitotriosidase activity (nmol/h.ml) } & \multirow[b]{2}{*}{$F$} & \multirow[b]{2}{*}{$P$} \\
\hline & $n$ & Mean & S.D. & Min-max & & \\
\hline $0^{*}$ & 94 & $10 \cdot 53$ & $3 \cdot 47$ & $3 \cdot 26-20 \cdot 83$ & $86 \cdot 660$ & $<0 \cdot 001$ \\
\hline 1 & 60 & $21 \cdot 94 \dagger$ & $16 \cdot 25$ & $5 \cdot 03-66 \cdot 92$ & & \\
\hline 2 & 234 & $30 \cdot 36+t$ & $15 \cdot 08$ & $6 \cdot 21-92 \cdot 40$ & & \\
\hline 3 & 207 & $42 \cdot 73 \dagger t s$ & $21 \cdot 29$ & $6 \cdot 94-119 \cdot 61$ & & \\
\hline 4 & 100 & $52 \cdot 04 \dagger+\delta \|$ & $24 \cdot 55$ & $9 \cdot 03-137 \cdot 16$ & & \\
\hline
\end{tabular}

* All healthy controls with DER $=0$.

$\dagger$ The least significant difference (LSD) pairwise comparison showed statistical significance compared with the score 0 group. $\$$ The LSD pairwise comparison showed statistical significance compared with the score 1 group.

$\S$ The LSD pairwise comparison showed statistical significance compared with the score 2 group.

|| The LSD pairwise comparison showed statistical significance compared with the score 3 group.

evaluate the effects of TB treatment should be further confirmed by follow-up studies, which are limited by potential difficulties, especially loss to follow-up. Second, the sample size calculation was performed after the sample collection. However, according to the method proposed by Dupont \& Plummer [26], the power, calculated using our sample size, was 0.964 , which suggests that our sample size had adequate power. Third, we did not consider the possible effects of sarcoidosis, fungi and nematode infections on enzyme activity in the controls. Nevertheless, these diseases are not prevalent in the general population, and are therefore unlikely to impact the results observed in our study.

In conclusion, our results suggest that chitotriosidase is a potential marker of the curative effects of TB treatment. Follow-up studies in various populations are needed in the future.

\section{ACKNOWLEDGEMENTS}

We thank our partners Dr Liqiong Bai (Hunan Institute of Tuberculosis Prevention and Treatment), 
and Ying Liang (School of Public Health, Central South University) for their input into this work.

This research received no specific grant from any funding agency, commercial or not-for-profit sectors.

\section{DECLARATION OF INTEREST}

None.

\section{REFERENCES}

1. WHO. Global Tuberculosis Control 2008: Surveillance, Planning, Financing. Geneva: WHO, 2008.

2. Horsburgh Jr. CR, et al. Revisiting rates of reactivation tuberculosis: a population-based approach. American Journal of Respiratory and Critical Care Medicine 2010; 182: 420-425.

3. Walzl G, et al. Immunological biomarkers of tuberculosis. Nature Reviews Immunology 2011; 11: 343-354.

4. WHO. Global Tuberculosis Report 2013. Geneva: WHO, 2013.

5. The Technical Guidance Group of the Fifth National TB Epidemiological Survey. The Fifth national tuberculosis epidemiological survey in 2010 [in Chinese]. Chinese Journal of Antituberculosis 2012; 34: 485-508.

6. WHO. Treatment of Tuberculosis: Guidelines, 4th edn. Geneva: WHO, 2010 (http://whqlibdoc.who.int/publications/2010/9789241547833_eng.pdf).

7. Di Rosa M, et al. Effect of interferon-gamma, interleukin-10, lipopolysaccharide and tumor necrosis factor-alpha on chitotriosidase synthesis in human macrophages. Clinical Chemistry and Laboratory Medicine 2005; 43: 499-502.

8. Malaguarnera $\mathbf{L}$, et al. Interferon-gamma, tumor necrosis factor-alpha, and lipopolysaccharide promote chitotriosidase gene expression in human macrophages. Journal of Clinical Laboratory Analysis 2005; 19: 128-132.

9. van Eijk M, et al. Characterization of human phagocyte-derived chitotriosidase, a component of innate immunity. International Immunology 2005; 17: 1505-1512.

10. Kologlu T, et al. Chitotriosidase as a possible marker of clinically evidenced atherosclerosis in dyslipidemic children. Journal of Pediatric Endocrinology \& Metabolism 2014; 27: 701-708.

11. Debono M, Gordee RS Antibiotics that inhibit fungal cell wall development. Annual Review of Microbiology 1994; 48: 471-497.
12. Bargagli E, et al. Chitotriosidase activity in the serum of patients with sarcoidosis and pulmonary tuberculosis. Respiration: International Review of Thoracic Diseases 2007; 74: 548-552.

13. Tasci $\mathbf{C}$, et al. Efficacy of serum chitotriosidase activity in early treatment of patients with active tuberculosis and a negative sputum smear. Therapeutics and Clinical Risk Management 2012; 8: 369-372.

14. Cakir G, et al. Serum chitotriosidase activity in pulmonary tuberculosis: response to treatment and correlations with clinical parameters. Annals of Laboratory Medicine 2012; 32: 184-189.

15. WHO. Treatment of Tuberculosis: Guidelines for National Programmes, 3rd edn. Geneva: WHO, 2003.

16. Chen M, et al. Gender and regional disparities of tuberculosis in Hunan, China. International Journal for Equity in Health 2014; 13: 32.

17. Deniz O, et al. Serum total cholesterol, HDL-C and LDL-C concentrations significantly correlate with the radiological extent of disease and the degree of smear positivity in patients with pulmonary tuberculosis. Clinical Biochemistry 2007; 40: 162-166.

18. Hollak CE, et al. Marked elevation of plasma chitotriosidase activity. A novel hallmark of Gaucher disease. Journal of Clinical Investigation 1994; 93: 1288-1292.

19. Korolenko TA, et al. Chitotriosidase as a marker of macrophage stimulation. Bulletin of Experimental Biology and Medicine 2000; 130: 948-950.

20. Guirado E, Schlesinger LS, Kaplan G. Macrophages in tuberculosis: friend or foe. Seminars in Immunopathology 2013; 35: 563-583.

21. Guan SP, et al. Chitinases: biomarkers for human diseases. Protein and peptide letters 2009; 16: 490-498.

22. Di Rosa M, et al. Modulation of chitotriosidase during macrophage differentiation. Cell Biochemistry and Biophysics 2013; 66: 239-247.

23. Ramanathan R, et al. Serum chitotriosidase, a putative marker of chronically activated macrophages, increases with normal aging. Journal of Gerontology, Series A, Biological Sciences and Medical Sciences 2013; 68: 1303-1309.

24. Harlander M, et al. Serial chitotriosidase measurements in sarcoidosis-two to five year follow-up study. Respiratory Medicine 2014; 108: 775-782.

25. Bouzas L, San Jose E, Tutor JC. Chitotriosidase activity in pleural effusions. Clinical Laboratory 2007; 53: 449-452.

26. Dupont WD, Plummer Jr. WD. Power and sample size calculations. A review and computer program. Controlled Clinical Trials 1990; 11: 116-128. 